\title{
GEOMETRY IN QUOTIENT REFLEXIVE SPACES
}

\author{
A. C. YORKE \\ (Received 10 April 1979) \\ Communicated by E. Strzelecki
}

\begin{abstract}
The structure and geometry of Banach spaces with the property that $E^{(4)}=\hat{E}^{* *}+E^{\perp \perp}$ are investigated: such spaces are called quotient reflexive spaces here. For these spaces, if $E$ is very smooth, $\hat{E}$ is also very smooth, and if $E^{*}$ is weakly locally uniformly rotund (WLUR), $E^{(4)}$ is smooth on a certain (relatively) norm dense subset of $\hat{E}^{* *}$. Consequently, for quotient reflexive spaces, WLUR and very-WLUR are equivalent in $E^{*}$.
\end{abstract}

1980 Mathematics subject classification (Amer. Math. Soc.): 46 B 99.

\section{Introduction and definitions}

Smith (1976) has recently given an example of a non-reflexive space with a smooth second dual. This has led to a great deal of interest in trying to find a variety of "smoothness" which will give reflexivity in second duals, but will not do so in first duals. The problem is still open.

One way to attack this problem is to investigate the smoothness of points of $\hat{E}^{* *}$, the embedding of $E^{* *}$ in the fourth dual $E^{(4)}$. This involves the study of the structure of higher duals, and, in particular, the study of spaces with the property that $E^{(4)}=\hat{E}^{* *}+E^{\perp \perp}$. Since this is equivalent to $E^{* *} / \hat{E}$ being reflexive (Lemma 1), these spaces will be referred to as quotient reflexive spaces. (Such spaces were called coreflexive spaces by Clarke (1972).)

Section 3 shows that, in quotient reflexive spaces, the geometry of $E$ and $E^{*}$ gives a great deal of information about smoothness at certain points of $E^{(4)}$. For example, if $x$ is a very smooth point of $E$, then $\hat{x}^{2}$ is a smooth point of $E^{(4)}$; if $E^{*}$ is weakly locally uniformly rotund (WLUR) at $f$, then $E^{(4)}$ is smooth at each

(c) 1983 Australian Mathematical Society 0263-6115/83 \$A2.00+0.00 
$\hat{F}_{f} \in \hat{E}^{* *}$ which attains its norm at $\hat{f}$. Consequently, any non-reflexive quotient reflexive space with a WLUR dual is smooth at each point of a (relatively) norm dense subset of $\hat{E}^{* *}$.

The rest of this section consists of definitions. For a real Banach space $E$, let $E^{(n)}$ denote its $n$th dual $\left(E^{(0)}=E\right)$. Let $S\left(E^{(n)}\right)$ denote the unit sphere of $E^{(n)}$, and $Q_{n}$ the natural embedding of the $n$th dual into the $(n+2)$ nd. The spaces $E^{(1)}, E^{(2)}, Q_{0} E, Q_{1} E^{(1)}, Q_{2} E^{(2)}, Q_{2} Q_{0} E$ and $Q_{3} Q_{1} E^{(1)}$ will usually be denoted by $E^{*}, E^{* *}, \hat{E}, \hat{E}^{*}, \hat{E}^{* *}, \hat{E}^{2}$, and $\hat{E}^{2 *}$, respectively.

The set valued mapping $D: E \rightarrow 2^{E^{*}}$ which associates with each $x \in E$ the $\left\{f \in E^{*}: f(x)=\|f\|\|x\|\right.$ and $\left.\|f\|=\|x\|\right\}$ is called the duality mapping. The duality mapping on $E^{(n)}$, for $n>0$, will be denoted by $D_{n}$. The sets $D(S(E))$ and $D_{n}\left(S\left(E^{(n)}\right)\right)$ will be denoted by $D(S)$ and $D_{n}(S)$, respectively. Elements of $D(x)$ will be denoted by $f_{x}$.

$E$ is smooth [very smooth] at $x \in S(E)$ if $D(x)\left[D_{2}(\hat{x})\right]$ is a singleton. $E$ is said to be smooth [very smooth] if $E$ is smooth [very smooth] at each $x \in S(E)$. $E$ is weakly locally uniformly rotund (WLUR) at $x \in S(E)$ if every sequence (or net) $\left\{F_{n}\right\}$ in $S\left(E^{* *}\right)$ with $\left\|F_{n}+\hat{x}\right\| \rightarrow 2$ has $\left(F_{n}-\hat{x}\right)(f) \rightarrow 0$ for all $f \in E^{*}$. This definition is equivalent to the usual definition (Yorke (1978; Lemma 2(1)). $E$ is very-WLUR at $x \in S(E)$ if every sequence (or net) $\left\{F_{n}\right\}$ in $S\left(E^{* *}\right)$ with $\| F_{n}+$ $\hat{x} \| \rightarrow 2$ has $\mathscr{F}\left(F_{n}-\hat{x}\right) \rightarrow 0$ for all $\mathscr{F} \in E^{(3)}$. $E$ is said to be WLUR [very-WLUR] if $E$ is WLUR [very-WLUR] at each $x \in S(E)$ (Yorke (1978)).

\section{The structure of higher duals}

Dixmier (1948) has shown that the third dual of a Banach space $E$ may be represented as $\hat{E}^{*}+E^{\perp}$, where $E^{\perp}=\operatorname{ker} Q_{0}^{*}$, and that the fourth dual has two distinct representations: $\hat{E}^{* *}+E^{* \perp}$ and $E^{\perp \perp}+E^{* \perp}$. Here $E^{* \perp}=\operatorname{ker} Q_{1}^{*}, E^{\perp \perp}$. is range of the linear isometry $Q_{0}^{* *}: E^{(2)} \rightarrow E^{(4)}$ and $\hat{E}^{* *} \cap E^{\perp \perp}=\hat{E}^{2}$. The elements of $E^{\perp}$ and $E^{* \perp}$ will be denoted by $x^{\perp}$ and $x^{* \perp}$, respectively. $E$ is said to be quasi-reflexive of order $n$ if $\hat{E}$ has codimension $n$ in $E^{* *}$ (Civin and Yood (1957)). James (1951) has constructed a quasi-reflexive space of order one: this is now usually referred to as the James space.

For the purpose of this paper a Banach space $E$ will be called quotient reflexive if $E^{* *} / \hat{E}$ is reflexive. Clearly, every quasi-reflexive space is quotient reflexive, and, in particular, the James space is quotient reflexive. However, not every space has this property (Davis and others (1974)).

In what follows the symbol " $\cong$ " will stand for "isometrically isomorphic to". 
LEMMA 1. The following are equivalent:

(1) $E$ is quotient reflexive;

(2) $E^{(4)}=\hat{E}^{* *}+E^{\perp \perp}$.

Proof. Applying Theorem 4.1(2) of Civin and Yood (1957; page 908) to $E^{* *}$ and $\hat{E}$ gives

$$
\frac{\left(E^{* *} / \hat{E}\right)^{* *}}{E^{* *} / \hat{E}} \cong \frac{E^{(4)}}{\hat{E}^{* *}+E^{\perp \perp}} .
$$

The result now follows. (Also, see Dixmier (1948; page 1070).)

The next lemma, which also appears in Clarke (1972; page 422), extends Corollary 4.2 of Civin and Yood (1957).

LemMa 2. Let $X$ be a closed subspace of $E$. Then

(1) $E$ is quotient reflexive if and only if $X$ and $E / X$ are quotient reflexive.

(2) $E$ is quotient reflexive if and only if $E^{*}$ is quotient reflexive.

Proof. Proceed as in Civin and Yood (1957; Theorem 4.1 and Lemma 3.4).

Now consider the space $Y=\hat{E}^{2 *}+E^{* * \perp} \subset E^{(5)}$. It is easy to check that, when $E$ is quotient reflexive, $Y$ is total over $E^{\perp \perp}$. Hence $Y$ and $E^{\perp \perp}$ are in duality, and the $\sigma\left(Y, E^{\perp \perp}\right)$ topology on $Y$ is compatible with this duality.

LEMMA 3. If $E$ is a quotient reflexive space, then $S\left(\hat{E}^{2 *}\right)$ is $\sigma\left(Y, E^{\perp \perp}\right)$ dense in $S(Y)$.

Proof. Let $S_{1}$ denote the $\sigma\left(Y, E^{\perp \perp}\right)$ closure of $S\left(\hat{E}^{2 *}\right)$ in $Y$, and assume there is a $y^{(5)} \in S(Y) \backslash S_{1}$. Since the $\sigma\left(Y, E^{\perp \perp}\right)$ topology separates points of $Y$, there must be a $Q_{0}^{* *} G \in E^{\perp \perp}$ and a real number $c>0$ such that $\left|x^{(5)}\left(Q_{0}^{* *} G\right)\right| \leqslant c$ for all $x^{(5)} \in S_{1}$ and $y^{(5)}\left(Q_{0}^{* *} G\right) \mid>c$. Now since $S\left(\hat{E}^{2 *}\right) \subset S_{1},\left|\hat{f}^{2 *}\left(Q_{0}^{* *} G\right)\right|=|G(f)|$ $\leqslant c$ for all $f \in S\left(E^{*}\right)$, so $\|G\|=\left\|Q_{0}^{* *} G\right\| \leqslant c$. Thus $\left|x^{(5)}\left(Q_{0}^{* *} G\right)\right| \leqslant c$ for all $x^{(5)} \in S(Y)$, and, in particular, $\left|\left(y^{(5)}\right)\left(Q_{0}^{* *} G\right)\right| \leqslant c$. Hence $S_{1}=S(Y)$.

\section{Geometry of quotient reflexive spaces}

A Banach space $E$ is Hahn-Banach smooth $(H-B)$ with respect to $f \in S\left(E^{*}\right)$ if $\left\|\hat{f}+x^{\perp}\right\|=\|f\|$ implies $x^{\perp}=0 . E$ is said to be $H-B$ smooth if $E$ is H-B smooth with respect to $f$ for all $f \in S\left(E^{*}\right)$. H-B smoothness was originally introduced by 
Sullivan (1976), and recently Smith and Sullivan (1977) have localized this concept. The localized version given here is distinct from Smith and Sullivan's, however.

Proposition 1. E is $H-B$ smooth with respect to $f \in S\left(E^{*}\right)$ if and only if the relative $\sigma\left(E^{*}, E\right)$ and $\sigma\left(E^{*}, E^{* *}\right)$ topologies on $S\left(E^{*}\right)$ agree at $f$.

Proof. Assume there is a net $\left\{f_{\alpha}\right\}$ in $S\left(E^{*}\right)$, an $F \in S\left(E^{* *}\right)$, and an $\varepsilon>0$ such that $f_{\alpha}(x) \rightarrow f(x)$ for all $x \in E$, but $\left|F\left(f_{\alpha}-f\right)\right| \geqslant \varepsilon$ for all $\alpha$. Let $\mathscr{F}$ be a $\sigma\left(E^{(3)}, E^{(2)}\right)$ cluster point of the $\left\{\hat{f}_{\alpha}\right\}:$ since $f_{\alpha}(x) \rightarrow f(x), \mathscr{F}$ must be of the form $\hat{f}+x^{\perp}$. But $1=\|f\| \leqslant\left\|\hat{f}+x^{\perp}\right\| \leqslant 1$, so $\|\mathscr{F}\|=1$. Thus $E$ cannot be H-B smooth with respect to $f$.

Conversely, assume there is an $\mathscr{F} \in S\left(E^{(3)}\right)$ with $\mathscr{F}=\hat{f}+x^{\perp},\|f\|=1$, and $x^{\perp} \neq 0$. Let $\left\{\hat{f}_{\alpha}\right\}$ be a net in $S\left(\hat{\mathrm{E}}^{*}\right)$ such that $\hat{f}_{\alpha}(F) \rightarrow \mathscr{F}(F)$ for all $F \in E^{* *}$. Clearly, $f_{\alpha}(x) \rightarrow f(x)$ for all $x \in E$, but $F\left(f_{\alpha}\right)$ cannot converge to $F(f)$ for all $F \in E^{* *}$ since $x^{\perp} \neq 0$. Thus the (relative) $\sigma\left(E^{*}, E\right)$ and $\sigma\left(E^{*}, E^{* *}\right)$ topologies on $S\left(E^{*}\right)$ do not agree at $f$.

Therefore, $\mathrm{H}-\mathrm{B}$ smoothness in $E$ gives information about $E^{*}$ as well as about $E^{(3)}$. The next result shows that, if $E$ is quotient reflexive, H-B smoothness also gives information about certain elements of $E^{(5)}$.

Proposition 2. Let $E$ be a quotient reflexive space. If $E$ is $H-B$ smooth with respect to $f \in S\left(E^{*}\right)$, then $\left\|\hat{f}^{2}+x^{* * \perp}\right\|=\|f\|$ implies $x^{* * \perp}=0$.

Proof. Let $y^{(5)}=\hat{f}^{2}+x^{* * \perp}$ be an element of $S(Y), Y=\hat{E}^{2 *}+E^{* * \perp}$, with $\|f\|=1$. Then, by Lemma 3 , there is a net $\left\{\hat{f}_{\alpha}^{2}\right\}$ in $S\left(\hat{E}^{2 *}\right)$ such that $\hat{f}_{\alpha}^{2}\left(Q_{0}^{* *} G\right)$ $\rightarrow y^{(5)}\left(Q_{0}^{* *} G\right)$ for all $Q_{0}^{* *} G \in E^{\perp \perp}$. Since $\hat{E}^{2} \subset E^{\perp \perp}, f_{\alpha}(x) \rightarrow f(x)$ for all $x \in E$. But $E$ is H-B smooth with respect to $f$, so, by Proposition $1, F\left(f_{\alpha}\right) \rightarrow F(f)$ for all $F \in E^{* *}$. Thus $\hat{f}_{\alpha}^{2}\left(\hat{F}+Q_{0}^{* *} G\right) \rightarrow \hat{f}^{2}\left(\hat{F}+Q_{0}^{* *} G\right)$ for all $Q_{0}^{* *} G \in E^{\perp \perp}$ and all $\hat{F} \in \hat{E}^{* *}$. Now since $E$ is quotient reflexive, $E^{(4)}=E^{\perp \perp}+\hat{E}^{* *}$, so $\hat{f}_{\alpha}^{2}\left(x^{(4)}\right) \rightarrow$ $\hat{f}^{2}\left(x^{(4)}\right)$ for all $x^{(4)} \in E^{(4)}$. Hence $x^{* * \perp}$ must be zero.

Proposition 3. Let $E$ be a quotient reflexive space. Then $E$ is very smooth at $x \in S(E)$ if and only if $E^{* *}$ is very smooth at $\hat{x} \in S(\hat{E})$.

Proof. It is sufficient to show that $E^{(4)}$ is smooth at $\hat{x}^{2}$. If $E$ is very smooth at $x, E^{* *}$ is smooth at $\hat{x}$, so $D_{2}(\hat{x})=\left\{\hat{f}_{x}\right\}$. Thus any $y^{(5)} \in D_{4}\left(\hat{x}^{2}\right)$ must be of the form $\hat{f}_{x}^{2}+x^{* * \perp}$. But if $E$ is very smooth at $x,\left\|\hat{f}_{x}+x^{\perp}\right\|=1$ implies $x^{\perp}=0$; that is, $E$ is H-B smooth with respect to $f_{x}$. Hence, by Proposition $2, x^{* * 1}=0$, so $D_{4}\left(\hat{x}^{2}\right)=\left\{\hat{f}_{x}^{2}\right\}$. 
Since $E$ is quotient reflexive if and only if $E^{* *}$ is quotient reflexive (Lemma 2(2)), using the methods of Proposition 3 one can show that $E^{(4)}$ is actually very smooth at $\hat{x}^{2}$.

Proposition 4. If $E^{*}$ is WLUR at $f \in S\left(E^{*}\right)$, then $E$ is $H-B$ smooth with respect to $f$.

Proof. Let $\left\{f_{\alpha}\right\}$ be any net in $S\left(E^{*}\right)$ which converges to $f$ in the $\sigma\left(E^{*}, E\right)$ topology. Since

$$
\left\|f_{\alpha}+f\right\|=\sup _{\|x\|=1}\left\{\left|\left(f_{\alpha}+f\right)(x)\right|\right\} \rightarrow \sup _{\|x\|=1}\{2|f(x)|\}=2,
$$

and $E^{*}$ is WLUR at $f,\left\{f_{\alpha}\right\}$ must converge to $f$ in the $\sigma\left(E^{*}, E^{* *}\right)$ topology. Proposition 1 now gives the result.

Proposition 5. Let $E$ be a quotient reflexive space. If $E$ is $W L U R$ at $f \in S\left(E^{*}\right)$, then $E^{* *}$ is very smooth at each $F_{f} \in D_{1}(f)$; that is, $E^{(4)}$ is smooth at each $\hat{F}_{f}$.

Proof. If $E^{*}$ is WLUR at $f$, then $E^{* *}$ is smooth at each $F_{f} \in D_{1}(f)$ (Yorke (1978; Theorem 1(1))). Thus any $y^{(5)} \in D_{4}\left(\hat{F}_{f}\right)$ must be of the form $\hat{f}^{2}+x^{* * 1}$. But, by Proposition 4, $E$ is H-B smooth with respect to $f$, so, by Proposition 2, $x^{* * \perp}=0$. Hence $D_{4}\left(\hat{F}_{f}\right)=\left\{\hat{f}^{2}\right\}$ and, since $F_{f} \in D_{1}(f)$ was chosen arbitrarily, the result follows.

Therefore, if $E$ is quotient reflexive, Proposition 5 and the Bishop-Phelps theorem (1961) show that, when $E^{*}$ is WLUR, $E^{(4)}$ is smooth at every point of a (relatively) norm dense subset of $S\left(\hat{E}^{* *}\right)$; or equivalently, $E^{* *}$. is very smooth at every point of $D_{1}(S)$.

In Yorke (1978) it was shown that if $E$ is very-WLUR, then $E^{*}$ is very smooth at certain points of $S\left(E^{*}\right)$. This leads to the next result.

Proposition 6. If $E$ is a quotient reflexive space, then $E^{*}$ is WLUR at $f \in S\left(E^{*}\right)$ if and only if $E^{*}$ is very-WLUR at $f$.

Proof. If $E^{*}$ is WLUR at $f$, then, by Proposition $5, E^{* *}$ is very smooth at each $F_{f} \in D_{1}(f)$. However, if $E^{* *}$ is very smooth at $F_{f}$ and $E^{*}$ is WLUR at $f$, then $E^{*}$ is very-WLUR at $f$ (Yorke (1978; Theorem 2(2))).

Thus WLUR and very-WLUR are equivalent in the dual of a quotient reflexive space. 
It is well known that if $E^{*}$ is very smooth; that is, if $\hat{E}^{*}$ is smooth, then $E$ must be reflexive. Smith (1976) has recently shown that the James space $J$ can be equivalently renormed so that $J^{*}$ is WLUR and $J^{* *}$ is smooth. Since $J$ is quasi-reflexive (of order one), Proposition 5 shows that $J^{* *}$ is actually very smooth on a norm dense subset of $S\left(J^{* *}\right)$. Thus the condition for reflexivity given above for first duals cannot be weakened for second duals.

\section{References}

E. Bishop and R. R. Phelps (1961), 'A proof that every Banach space is subreflexive', Bull. Amer. Math. Soc. 67, 97-98.

P. Civin and B. Yood (1957), 'Quasi-reflexive spaces', Proc. Amer. Math. Soc. 8, 906-911.

J. R. Clarke (1972), 'Coreflexive and somewhat reflexive Banach spaces', Proc. Amer. Math. Soc. 36, 421-427.

W. J. Davis, T. Figiel, W. B. Johnson and A. Pelczynski (1971), 'Factoring weakly compact operators', J. Functional Analysis 17, 311-327.

J. Dixmier (1948), 'Sur un théorème de Banach', Duke Math.J. 15, 1057-107I.

R. C. James (1951), 'A non-reflexive Banach space isometric with its second conjugate space', Proc. Nat. Acad. Sci. U.S.A. 37, 174-177.

M. A. Smith (1976), 'A smooth, non-reflexive second conjugate space', Bull. Austral. Math. Soc. 15, 29-31.

M. A. Smith and F. Sullivan (1977), 'Extremely smooth Banach spaces', preprint.

F. Sullivan (1976), 'Some geometric properties of higher duals in Banach spaces', Illinois J. Math. 21, 315-331.

A. C. Yorke (1978), 'Differentiability and local rotundity', J. Austral. Math. Soc. Ser. A 28, 205-213.

\section{Department of Mathematics}

The University of Western Australia

Nedlands, W. A. 6009

Australia 